\title{
Instant-Form and Light-Front Hamiltonian and Path Integral Formulations of the Conformally Gauge-Fixed Polyakov D1-Brane Action in the Presence of a Scalar Axion Field and an $U(1)$ Gauge Field ${ }^{*}$
}

\author{
Usha Kulshreshtha" ${ }^{1 \#}$, Daya Shankar Kulshreshtha \\ ${ }^{1}$ Department of Physics, Kirori Mal College, University of Delhi, Delhi, India. \\ ${ }^{2}$ Department of Physics and Astrophysics, University of Delhi, Delhi, India \\ Email: " ushakulsh@gmail.com, dskulsh@gmail.com
}

Received February 9, 2013; revised March 13, 2013; accepted March 25, 2013

Copyright (C) 2013 Usha Kulshreshtha, Daya Shankar Kulshreshtha. This is an open access article distributed under the Creative Commons Attribution License, which permits unrestricted use, distribution, and reproduction in any medium, provided the original work is properly cited.

\begin{abstract}
Recently we have studied the instant-form quantization (IFQ) and the light-front quantization (LFQ) of the conformally gauge-fixed Polyakov D1 brane action using the Hamiltonian and path integral formulations. The IFQ is studied in the equal world-sheet time framework on the hyperplanes defined by the world-sheet time $\sigma^{0}=\tau=$ constant and the LFQ in the equal light-cone world-sheet time framework, on the hyperplanes of the light-front defined by the light-cone world-sheet time $\sigma^{+}=(\tau+\sigma)=$ constant. The light-front theory is seen to be a constrained system in the sense of Dirac in contrast to the instant-form theory. However, owing to the gauge anomalous nature of these theories, both of these theories are seen to lack the usual string gauge symmetries defined by the world-sheet reparametrization invariance (WSRI) and the Weyl invariance (WI). In the present work we show that these theories when considered in the presence of background gauge fields such as the NSNS 2-form gauge field $B_{\alpha \beta}(\sigma, \tau)$ or in the presence of $U(1)$ gauge field $A_{\alpha}(\sigma, \tau)$ and the constant scalar axion field $C(\sigma, \tau)$, then they are seen to possess the usual string gauge symmetries (WSRI and WI). In fact, these background gauge fields are seen to behave as the Wess-Zumino or Stueckelberg fields and the terms containing these fields are seen to behave as Wess-Zumino or Stueckelberg terms for these theories.
\end{abstract}

Keywords: Lagrangian and Hamiltonian Approach; Hamiltonian Quantization; Path Integral Quantization; Light-Front Quantization; Theory of Quantized Fields; Constrained Dynamics; D-Brane Actions; Polyakov Action; Strings and Branes; String Gauge Symmetry; Gauge Field Theories

\section{Introduction}

Study of D-brane actions is a domain of wider interest [1-20] in string theories. Polyakov D-brane action [18,12-20] does not involve any square root [1-11] and is in particular, simpler to study. Recently, we have studied the instant-form (IF) quantization (IFQ) of this action [12] for the D1 brane in the conformal gauge (CG), using the

\footnotetext{
*Part of this work was presented by DSK as an Invited Talk at "International Conference on Light-Cone Physics LC2011: Applications of Light-Cone Coordinates to Highly Relativistic Systems”, held at the Southern Methodist University, Dallas, Texas, May 22-27, 2011.

${ }^{*}$ Corresponding author.
}

Hamiltonian [21] and path integral [22-25] formulations in the instant-form (IF) of dynamics (on the hyperplanes defined by the world-sheet (WS) time $\sigma^{0}=\tau=$ costant ) [26,27]. We have also studied its LFQ [13-20] using the light-front (LF) dynamics (on the hyperplanes of the LF defined by the light-cone (LC) WS time $\sigma^{+}=(\tau+\sigma)=$ constant $)$ [26-33].

The LF theory [13-20] is seen to be a constrained system in the sense of Dirac [21], which is in contrast to the corresponding IF theory [12], where the theory remains unconstrained in the sense of Dirac [21]. The LF theory is seen to possess a set of twenty six second-class 
contraints [13-20]. Further, the conformally gauge-fixed Polyakov D1 brane action (CGFPD1BA) describing a gauge-noninvariant (GNI) theory (being a gauge-fixed theory) is seen to describe a gauge-invariant (GI) theory in the presence of an antisymmetric NSNS 2-form gauge field $B_{\alpha \beta}(\tau, \sigma)$ [13-20].

Recently we have shown [13-20] that this NSNS 2-form gauge field behaves like a Wess-Zumino (WZ) field and the term involving this field behaves like a WZ term for the CGFPD1BA [13-20]. We have also studied the Hamiltonian and path integral formulations of the CGFPD1BA with and without a scalar dilaton field in the IF [12] as well as in the LF [13-18] dynamics. In both the above cases the theory is seen (as expected) to be gaugenoninvariant (GNI), possessing a set of second-class constraints in each case, owing to the conformal gaugefixing $[1-8,12-20]$ of the theory.

The CGFPD1BA being GNI does not respect the usual string gauge symmetries defined by the world-sheet (WS) reparametrization invariance (WSRI) and the Weyl invariance (WI) [1-8,12-20]. However, in the presence of a constant 2-form gauge field $B_{\alpha \beta}$ it is seen [13] to describe a gauge-inavriant (GI) theory [13-20] respecting the usual string gauge symmetries defined by the WSRI and the WI.

The IF and the LF Hamiltonian and path integral formulations of the CGFPD1BA have been studied by us in Refs. [12-18]. The IF and the LF Hamiltonian and path integral formulations of this theory in the presence of the constant 2-form gauge field $B_{\alpha \beta}$ have been studied by us in Ref. [13].

The question of the string gauge symmetries associated with the Polyakov D1 brane action in the presence of some other background fields such as the $U(1)$ gauge field $A^{\mu}(\tau, \sigma)$ and the constant scalar axion field $C(\tau, \sigma)$ have been considered by us in Ref. [19].

In the present work, we study the CGFPD1BA in the presence of some other background fields such as the $U(1)$ gauge field $A^{\mu}(\tau, \sigma)$ and the constant scalar axion field $C(\tau, \sigma)$

In the next section we recap the basic essentials of the CGFPD1BA in IFQ as well as in th LF quantization (LFQ) [12-18]. In Section 3, we recap the basic essentials of this theory in the presence of the constant NSNS 2 -form gauge field $B_{\alpha \beta}$ [13]. In Section 4, we study the IFQ [26,27] as well as the LFQ [26-33] of the CGFPD1BA in the presence of some other background fields such as the $U(1)$ gauge field $A^{\mu}(\tau, \sigma)$ and the constant scalar axion field $C(\tau, \sigma)[19,20]$. Finally the summary and discussion is given in Section 5 .

\section{Conformally Gauge-Fixed Polyakov D1 Brane Action (CGFPD1BA): A Recap}

The Polyakov D1 brane action in a d-dimensional curved background $h_{\alpha \beta}$ is defined by [1-8,12-20]:

$$
\begin{aligned}
& \tilde{S}=\int \tilde{\mathcal{L}} \mathrm{d}^{2} \sigma \\
& \mathcal{L}=\left[\frac{-T}{2} \sqrt{-h} h^{\alpha \beta} G_{\alpha \beta}\right] \\
& h=\operatorname{det}\left(h_{\alpha \beta}\right), G_{\alpha \beta}=\partial_{\alpha} X^{\mu} \partial_{\beta} X^{v} \eta_{\mu v} \\
& \eta_{\mu v}=\operatorname{diag}(-1,+1, \cdots,+1) \\
& \mu, v=0,1, i ., i=2,3, \cdots,(d-1), \alpha, \beta=01(I F Q) \\
& \mu, v=+,-, i ., i=2,3, \cdots,(d-1), \alpha, \beta=+,-(I F Q)
\end{aligned}
$$

Here $\sigma^{\alpha} \equiv(\tau, \sigma)$ are the two parameters describing the worldsheet (WS). The overdots and primes would denote the derivatives with respect to $\tau$ and $\sigma . T$ is the string tension. $G_{\alpha \beta}$ is the induced metric on the WS and $X^{\mu}(\tau, \sigma)$ are the maps of the WS into the $d$ dimensional Minkowski space and describe the strings evolution in space-time [1-8,12-20].

Here $h_{\alpha \beta}$ are the auxiliary fields (which turn out to be proportional to the metric tensor $\eta_{\alpha \beta}$ of the twodimensional surface swept out by the string). One can think of $\tilde{S}$ as the action describing d-massless scalar fields $X^{\mu}$ in two-dimensions moving on a curved background $h_{\alpha \beta}$.

Also because the metric components $h_{\alpha \beta}$ are varied in the above equation, the 2-dimensional gravitational field $h_{\alpha \beta}$ is treated not as a given background field, but rather as an adjustable quantity coupled to the scalar fields.

The above action has three local gauge symmetries given by the 2-dimensional (2D) WSRI and WI as follows:

$$
\begin{aligned}
& X^{\mu} \rightarrow \tilde{X}^{\mu}=\left[X^{\mu}+\delta X^{\mu}\right] \\
& \delta X^{\mu}=\left[\zeta^{\alpha}\left(\partial_{\alpha} X^{\mu}\right)\right] \\
& h^{\alpha \beta} \rightarrow \tilde{h}^{\alpha \beta}=\left[h^{\alpha \beta}+\delta h^{\alpha \beta}\right] \\
& \delta h^{\alpha \beta}=\left[\zeta^{\gamma} \partial_{\gamma} h^{\alpha \beta}-\partial_{\gamma} \zeta^{\alpha} h^{\gamma \beta}-\partial_{\gamma} \zeta^{\beta} h^{\alpha \gamma}\right] \\
& h_{\alpha \beta} \rightarrow[\Omega] h_{\alpha \beta}, \Omega(\tau, \sigma)=\exp (2 \omega), \omega=\omega(\tau, \sigma)
\end{aligned}
$$

where the WSRI is defined for the two parameters $\zeta^{\alpha} \equiv \zeta^{\alpha}(\tau, \sigma)$; and the WI and is specified by a function $\Omega \equiv \Omega(\tau, \sigma)$. In the following we would, however, work in the so-called orthonormal gauge where one sets $\Omega=1$.

Also for the CGFPD1BA one makes use of the fact that the 2D metric $h_{\alpha \beta}$ is also specified by three independent functions as it is a symmetric $2 \times 2$ metric. One can therefore use these gauge symmetries of the theory to 
choose $h_{\alpha \beta}$ to be of a particular form in the IFQ (on the hyperplanes defined by $x^{0}=t=$ constant ) as follows:

$$
h_{\alpha \beta}:=\eta_{\alpha \beta}, h^{\alpha \beta}:=\eta^{\alpha \beta}
$$

In the IFQ we take

$$
\begin{gathered}
h_{\alpha \beta}=\eta_{\alpha \beta}=\left(\begin{array}{ll}
-1 & 0 \\
0 & +1
\end{array}\right) \\
h^{\alpha \beta}=\eta^{\alpha \beta}=\left(\begin{array}{ll}
-1 & 0 \\
0 & +1
\end{array}\right)
\end{gathered}
$$

with

$$
\sqrt{-h}=\sqrt{-\operatorname{det}\left(h_{\alpha \beta}\right)}=+1
$$
by:

In LFQ we use the Light-Cone (LC) variables defined

$$
\sigma^{ \pm}:=(\tau \pm \sigma) ; X^{ \pm}:=\left(X^{0} \pm X^{1}\right) / \sqrt{2}
$$

In the LFQ (on the hyperplanes defined by $x^{+}=\tau=$ constant ) we take:

$$
\begin{aligned}
& h_{\alpha \beta}:=\eta_{\alpha \beta}=\left(\begin{array}{ll}
0 & -1 / 2 \\
-1 / 2 & 0
\end{array}\right) \\
& h^{\alpha \beta}:=\eta^{\alpha \beta}=\left(\begin{array}{ll}
0 & -2 \\
-2 & 0
\end{array}\right)
\end{aligned}
$$

with

$$
\sqrt{-h}=\sqrt{-\operatorname{det} h_{(\alpha \beta)}}=+\frac{1}{2}
$$

The action $\tilde{S}$ in the CG (in IFQ as well as in LFQ) finally becomes: reads [1-8,12-20]:

$$
\begin{aligned}
& S^{N}=\int \mathcal{L}^{N} \mathrm{~d}^{2} \sigma \\
& \mathcal{L}^{N}=\left(\frac{-T}{2}\right)\left[\partial^{\beta} X^{\mu} \partial_{\beta} X_{\mu}\right] \\
& \beta=0,1, \mu=0,1, i, i=2,3, \cdots, 25(I F Q) \\
& \beta=+,-, \mu=+,-, i ; i=2,3, \cdots, 25(L F Q)
\end{aligned}
$$

This is the CGFPD1BA. In the IFQ it reads:

$$
\begin{aligned}
& S_{1}=\int L_{1} \mathrm{~d}^{2} \sigma, \mathcal{L}_{1}=\frac{-T}{2}\left[\partial_{\sigma} X^{\mu} \partial_{\sigma} X_{\mu}-\partial_{\tau} X^{\mu} \partial_{\tau} X_{\mu}\right] \\
& \mathcal{L}_{1}=\frac{-T}{2}\left[\left(X^{\prime}\right)^{2}-(\dot{X})^{2}\right] \\
& \partial_{\tau} \equiv \frac{\partial}{\partial \tau}, \partial_{\sigma} \equiv \frac{\partial}{\partial \tau}, \dot{X}^{\mu} \equiv \frac{\partial X^{\mu}}{\partial \tau}, X^{\prime \mu}=\frac{\partial X^{\mu}}{\partial \sigma}
\end{aligned}
$$

The IFQ of this action has been studied by us recently [12] and we recap it here very briefly. The canonical momenta conjugate to $X^{\mu}$ obtained from the above action are:

$$
P^{\mu}:=\frac{\partial \mathcal{L}_{1}}{\partial\left(\partial_{\tau} X^{\mu}\right)}=T \partial_{\tau} X^{\mu}
$$

Here the velocities $\partial_{\tau} X^{\mu}=\frac{1}{T} P^{\mu}$ are expressible. Canonical Hamiltonian density for the above theory is:

$$
\mathcal{H}_{1}^{c}=\left[P^{\mu}\left(\partial_{\tau} X_{\mu}\right)-\mathcal{L}_{1}\right]=\left[\frac{1}{2 T} P^{2}+\frac{T}{2}\left(X^{\prime}\right)^{2}\right] ;
$$

The quantization of the system is trivial. The nonvanishing equal WS-time (EWST) commutation relations (CR's) for this theory are obtained as:

$$
\left[X^{\mu}(\sigma, \tau), P_{v}\left(\sigma^{\prime}, \tau\right)\right]=i \delta_{v}^{\mu} \delta\left(\sigma-\sigma^{\prime}\right)
$$

where $\delta\left(\sigma-\sigma^{\prime}\right)$ is the one-dimensional Dirac distribution function.

It is obvious from the above considerations that the above theory is unconstrained in the sense of Dirac [21]. It may be important to emphasize here that an unconstrained system like the above theory is a gauge-noninvariant theory and it is some what akin to a gauge-fixed gauge-invariant theory which makes it a gauge-noninvariant system. In the presence of a scalar dilaton field the theory of course, becomes a constrained system in the sense of Dirac as shown in our earlier work [12]. For further details of the IFQ of this theory we refer to our earleir work of Ref. [12].

The CGFPD1BA in the LFQ reads [12-18]:

$$
\begin{aligned}
& S_{2}=\int \mathcal{L}_{2} \mathrm{~d} \sigma^{+} \mathrm{d} \sigma^{-} \\
& \mathcal{L}_{2}=\left[\frac{-T}{2}\right] \\
& \cdot\left[\left(\partial_{+} X^{+}\right)\left(\partial_{-} X^{-}\right)+\left(\partial_{+} X^{-}\right)\left(\partial_{-} X^{+}\right)+\left(\partial_{+} X^{i}\right)\left(\partial_{-} X^{i}\right)\right] \\
& \mu, v=+,-, i, i=2,3, \cdots, 25
\end{aligned}
$$

The canonical momenta $P^{+}, P^{-}$and $P_{i}$ canonically conjugate respectively to $X^{-}, X^{+}$and $X^{i}$, obtained from the above LF action are:

$$
\begin{gathered}
P^{+}:=\frac{\partial \mathcal{L}_{2}}{\partial\left(\partial_{+} X^{-}\right)}=-\frac{T}{2}\left(\partial_{-} X^{+}\right) \\
P^{-}:=\frac{\partial \mathcal{L}_{2}}{\partial\left(\partial_{+} X^{+}\right)}=-\frac{T}{2}\left(\partial_{-} X^{-}\right) \\
P_{i}:=\frac{\partial \mathcal{L}_{2}}{\partial\left(\partial_{+} X^{i}\right)}=-\frac{T}{2}\left(\partial_{-} X^{i}\right)
\end{gathered}
$$

Above equations however, imply that the theory possesses 26 primary constraints:

$$
\rho_{1}=\left(P^{+}+\frac{T}{2} \partial_{-} X^{+}\right) \approx 0
$$




$$
\begin{gathered}
\rho_{2}=\left(P^{-}+\frac{T}{2} \partial_{-} X^{-}\right) \approx 0 \\
\rho_{i}=\left(P_{i}+\frac{T}{2} \partial_{-} X^{i}\right) \approx 0, i=2,3, \cdots, 25 .
\end{gathered}
$$

Canonical Hamiltonian density for the above theory is:

$$
\begin{aligned}
\mathcal{H}_{2}^{c} & =\left[P^{+}\left(\partial_{+} X^{-}\right)+P^{-}\left(\partial_{+} X^{+}\right)+P_{i}\left(\partial_{+} X^{i}\right)-\mathcal{L}_{2}\right] \\
& \approx 0
\end{aligned}
$$

After including the above 26 primary constraints of the theory in the canonical Hamiltonian density $\mathcal{H}_{2}^{c}$ with the help of Lagrange multiplier fields $u, v$ and $w_{i}$, the total Hamiltonian density $\mathcal{H}_{2}^{T}$ could be written as

$$
\begin{aligned}
\mathcal{H}_{2}^{T} & =\left[u\left(P^{+}+\frac{T}{2} \partial_{-} X^{+}\right)+v\left(P^{-}+\frac{T}{2} \partial_{-} X^{-}\right)\right. \\
& \left.+w_{i}\left(P_{i}+\frac{T}{2} \partial_{-} X^{i}\right)\right]
\end{aligned}
$$

We treat $u, v$ and $w_{i}$ as dynamical. The Hamiltons equations obtained from the total Hamiltonian

$H_{2}^{T}=\int \mathcal{H}_{2}^{T} \mathrm{~d} \sigma^{-}$, are the equations of motion of the theory that preserve the constraints of the theory in the course of time. Demanding that these primary constraints of the theory be preserved in the course of time one does not get any secondary constraints. The theory is thus seen to possess only 26 above constraints. Further, the matrix of the Poisson brackets of the above constraints is seen to be nonsingular, implying that the set of these constraints is second-class and consequently the theory is GNI [12-18]. The theory indeed does not possess the usual local string gauge symmetries defined by the WSRI and the WI [12-18].

This action is thus seen to lack the local gauge symmetries. This is in contrast to the fact that the original action $\tilde{S}$ had the local gauge symmetries and was therefore GI. The theory defined by the action $S^{N}$, on the other hand describes a GNI theory. This is not surprising at all because the theory defined by $S^{N}$ is afterall (conformally) a gauge-fixed theory and consequently it is not expected to be GI anyway.

Infact, the IF theory defined by $S^{N}$ is seen to be unconstrained [12] whereas the LF theory is seen to possess a set of 26 second-class constraints [12-18]. In both the cases theory does not respect the usual local string gauge symmetries defined by WSRI and WI [12-18].

\section{CGFPD1BA in the Presence of a 2-Form Gauge Field $B_{\alpha \beta}$ : A Recap}

We now consider this CGFPD1BA in the presence of a constant background antisymmetric 2-form NSNS gauge field $B_{\alpha \beta}$ studied earlier by Schmidhuber, de Alwis and
Sato, Tseytlin and Abou Zeid and Hull and others defined by [1-8]. This theory has been studied by us earlier in Ref. [13]. This theory is defined by the action [1-8,13]:

$$
\begin{aligned}
& S_{B}^{I}=\int \mathcal{L}_{B}^{I} \mathrm{~d}^{2} \sigma \\
& \mathcal{L}_{B}^{I}=\left[\mathcal{L}^{C}+\mathcal{L}^{B}\right] \\
& \mathcal{L}^{C}=\left[\lambda \mathcal{L}^{N}\right]=\left(\frac{-T}{2}\right)\left[\lambda \partial^{\beta} X^{\mu} \partial_{\beta} X_{\mu}\right] \\
& \mathcal{L}^{B}=\left(\frac{-T}{2}\right)\left[\Lambda \varepsilon^{\alpha \beta} B_{\alpha \beta}\right] \\
& \lambda=\sqrt{\left(1+\Lambda^{2}\right)}, \Lambda=\mathrm{constant}, \varepsilon^{\alpha \beta}=\left(\begin{array}{cc}
0 & 1 \\
-1 & 0
\end{array}\right) \\
& B_{\alpha \beta}:=\partial_{\alpha} X^{\mu} \partial_{\beta} X^{v} B_{\mu \nu}, B_{\alpha \beta}=\left(\begin{array}{ll}
0 & B \\
-B & 0
\end{array}\right) \\
& B=B_{01}=-B_{10}(I F Q) \\
& B=B_{+-}=-B_{-+}(L F Q) \\
& \alpha, \beta=0,1, \mu=0,1, i, i=2,3, \cdots, 25(I F Q) \\
& \alpha, \beta=+,-, \mu=+,-, i, i=2,3, \cdots, 25(L F Q)
\end{aligned}
$$

It is important to recollect here that the 2-form gauge field $B_{\alpha \beta}$ is a scalar field in the target-space whereas it is a constant anti-symmetric tensor field in the worldsheet space. The above action is seen to be GI in the IFQ as well as in the LFQ [13]. It is seen to possess only one constraint in IFQ and a set of 27 constraints in LFQ. The nature of constraints in both the cases is seen to be first-class implying that the theory is GI in both the cases [13]. In Ref. [13], we have studied the Hamiltonian and path integral formulations of this theory under appropriate gauge-fixing.

\subsection{Instant-Form Quantization}

In the following, we study the IFQ of this above theory using the EWST framework of dynamics on the hyperplanes defined by the WS-time $\sigma^{0}=\tau=$ constant [26, 27]. In the IFQ, the above action reads as:

$$
S_{3}=\int \mathcal{L}_{3} \mathrm{~d} \tau \mathrm{d} \sigma, \mathcal{L}_{3}=\left[-\frac{\lambda T}{2}\left[\left(X^{\prime}\right)^{2}-(\dot{X})^{2}\right]-\Lambda T B\right]
$$

Canonical momenta are

$$
P^{\mu}:=\frac{\partial \mathcal{L}_{3}}{\partial\left(\partial_{\tau} X_{\mu}\right)}=\left[\lambda T \dot{X}^{\mu}\right], \Pi_{B}:=\frac{\partial \mathcal{L}_{3}}{\partial\left(\partial_{\tau} B\right)}=0
$$

Here $P^{\mu}$ and $\Pi_{B}$ are the canonical momenta conjugate respectively to $X_{\mu}$ and $B\left(\equiv B_{01}=-B_{10}\right)$. The above theory is thus seen to possess one primary constraint: $\Phi_{1}=\Pi_{B} \approx 0$. Canonical Hamiltonian density of 
this theory is:

$$
\begin{aligned}
& \mathcal{H}_{3}^{c}=\left[P^{\mu}\left(\partial_{\tau} X_{\mu}\right)+\Pi_{B}\left(\partial_{\tau} B\right)-\mathcal{L}_{3}\right] \\
& \mathcal{H}_{3}^{c}=\left[\frac{1}{2 \lambda T} P^{\mu} P_{\mu}+\frac{\lambda T}{2}\left(X^{\prime}\right)^{2}+\Lambda T B\right]
\end{aligned}
$$

After incorporating the primary constraint of the theory in the canonical Hamiltonian density with the help of Lagrange multiplier field $u(\tau, \sigma)$ (to be treated as dynamical) the total Hamiltonian density of the theory could be written as:

$$
\mathcal{H}_{3}^{T}=\left[\frac{1}{2 \lambda T} P^{\mu} P_{\mu}+\frac{\lambda T}{2}\left(X^{\prime}\right)^{2}+\Lambda T B+u \Pi_{B}\right]
$$

Also, the momenta canonically conjugate to $\mu$ is denoted by $p_{\mu}$.

The Hamiltons equations obtained from the total Hamiltonian: $H_{3}^{T}=\int \mathcal{H}_{3}^{T} \mathrm{~d} \sigma$ are the equations of motion that preserve the constraints of the theory in the course of time. Demanding that the primary constraint of the theory be preserved in the course of time one does not get any further constraints. The theory is thus seen to posses the only one above constraint.

The Poission bracket of the constraint of the theory with itself is seen to be zero implying that the constraint is first-class and that the theory is GI. It is indeed seen to posses three local gauge symmetries given by the 2D WSRI and the WI defined by:

$$
\begin{aligned}
& X^{\mu} \rightarrow \tilde{X}^{\mu}=\left[X^{\mu}+\delta X^{\mu}\right] \\
& \delta X^{\mu}=\left[\zeta^{\alpha}\left(\partial_{\alpha} X^{\mu}\right)\right] \\
& h^{\alpha \beta} \rightarrow \tilde{h}^{\alpha \beta}=\left[h^{\alpha \beta}+\delta h^{\alpha \beta}\right] \\
& \delta h^{\alpha \beta}=\left[\zeta^{\gamma} \partial_{\gamma} h^{\alpha \beta}-\partial_{\gamma} \zeta^{\alpha} h^{\gamma \beta}-\partial_{\gamma} \zeta^{\beta} h^{\alpha \gamma}\right] \\
& B_{\alpha \beta} \rightarrow \tilde{B}_{\alpha \beta}=\left[B_{\alpha \beta}+\delta B_{\alpha \beta}\right] \\
& \delta B_{\alpha \beta}=\left[\zeta^{\alpha} \partial_{\alpha} B_{\alpha \beta}\right] \\
& h_{\alpha \beta} \rightarrow \Omega\left[h_{\alpha \beta}\right], \Omega=\exp (2 \omega)
\end{aligned}
$$

It is important to recollect here that the 2-form gauge field $B_{\alpha \beta}$ is a scalar field in the target-space whereas it is a constant anti-symmetric tensor field in the worldsheet space (and consequently we have $\delta B_{\alpha \beta}=0$ ).

Thus the theory is seen to be GI in IFQ as well as in LFQ. It is therefore gauge-nonanomalous possessing the three local gauge symmetries defined by the 2D WSRI and the WI. The theory could therefore be quantized under appropriate gauge-fixing.

Hamiltonian and path integral formulations of this theory could be studied under appropriate gauge-fixing e.g., under the gauge: $\Phi=B \approx 0$. Corresponding to this choice of gauge the total set of constraints of the theory under which the quantization of the theory could e.g., be studied becomes: $\Phi_{1}=\Pi_{B} \approx 0$ and $\Phi_{2}=\Phi=B \approx 0$. The matrix of the Poisson brackets of these constraints is seen to be nonsingular implying that the corresponding set of constraints is second-class. Following the Dirac quantization procedure in the Hamiltonian formulation, the nonvanishing EWST CR's of the theory under the above gauge are obtained as:

$$
\left[X^{\mu}(\sigma, \tau), P^{v}\left(\sigma^{\prime}, \tau\right)\right]=(i) \eta^{\mu v} \delta\left(\sigma-\sigma^{\prime}\right)
$$

\subsection{Light-Front Quantization}

In LFQ, using the ELCWST framework of dynamics on the hyperplanes defined by the LC WS-time $\sigma^{+}=(\tau+\sigma)=$ constant, the action of the theory reads:

$$
\begin{aligned}
S_{4} & =\int \mathcal{L}_{4} \mathrm{~d} \sigma^{+} \mathrm{d} \sigma^{-} \\
\mathcal{L}_{4} & =\left[-\frac{\lambda T}{2}\right]\left[\left(\partial_{+} X^{+}\right)\left(\partial_{-} X^{-}\right)+\left(\partial_{+} X^{-}\right)\left(\partial_{-} X^{+}\right)\right. \\
& \left.+\left(\partial_{+} X^{i}\right)\left(\partial_{-} X^{i}\right)-\Lambda T B\right]
\end{aligned}
$$

Canonical momenta $\Pi_{B}, P^{+}, P^{-}$, and $P_{i}$ conjugate respectively to $B, X^{-}, X^{+}$, and $X_{i}$ are:

$$
\begin{aligned}
\Pi_{B} & :=\frac{\partial \mathcal{L}_{4}}{\partial\left(\partial_{+} B\right)}=0 \\
P^{+} & :=\frac{\partial \mathcal{L}_{4}}{\partial\left(\partial_{+} X^{-}\right)}=-\frac{\lambda T}{2}\left(\partial_{-} X^{+}\right) \\
P^{-} & :=\frac{\partial \mathcal{L}_{4}}{\partial\left(\partial_{+} X^{+}\right)}=-\frac{\lambda T}{2}\left(\partial_{-} X^{-}\right) \\
P_{i} & :=\frac{\partial \mathcal{L}_{4}}{\partial\left(\partial_{+} X^{i}\right)}=-\frac{\lambda T}{2}\left(\partial_{-} X^{i}\right), i=2,3, \cdots, 25
\end{aligned}
$$

Above equations however, imply that the theory possesses the following 27 primary constraints:

$$
\begin{aligned}
& \psi_{1}=\Pi_{B} \approx 0 \\
& \psi_{2}=\left[P^{+}+\frac{\lambda T}{2}\left(\partial_{-} X^{+}\right)\right] \approx 0 \\
& \psi_{3}=\left[P^{-}+\frac{\lambda T}{2}\left(\partial_{-} X^{-}\right)\right] \approx 0 \\
& \psi_{i}=\left[P_{i}+\frac{\lambda T}{2}\left(\partial_{-} X^{i}\right)\right] \approx 0, i=2,3, \cdots, 25
\end{aligned}
$$

Canonical Hamiltonian density corresponding to $\mathcal{L}_{4}$ is

$$
\begin{aligned}
\mathcal{H}_{4}^{c}= & {\left[\Pi_{B}\left(\partial_{+} B\right)+P^{+}\left(\partial_{+} X^{-}\right)\right.} \\
& \left.+P^{-}\left(\partial_{+} X^{+}\right)+P_{i}\left(\partial_{+} X^{i}\right)-\mathcal{L}_{4}\right]
\end{aligned}
$$




$$
\mathcal{H}_{4}^{c}=[\Lambda T B]
$$

After including the primary constraints of the theory in the canonical Hamiltonian density $\mathcal{H}_{4}^{c}$ with the help of Lagrange multiplier fields $s\left(\sigma^{+}, \sigma^{-}\right), u\left(\sigma^{+}, \sigma^{-}\right)$, $v\left(\sigma^{+}, \sigma^{-}\right)$and $w_{i}\left(\sigma^{+}, \sigma^{-}\right)$(which we treat as dynamical), the total Hamiltonian density of the theory could be written as:

$$
\begin{aligned}
\mathcal{H}_{4}^{T} & =\left[\mathcal{H}_{2}^{c}+s \psi_{1}+u \psi_{2}+v \psi_{3}+w_{i} \psi_{i}\right] \\
\mathcal{H}_{4}^{T} & =\left[\Lambda T B+s \Pi_{B}+u\left[P^{+}+\left(\frac{\lambda T}{2}\right)\left(\partial_{-} X^{+}\right)\right]\right. \\
& +v\left[P^{-}+\left(\frac{\lambda T}{2}\right)\left(\partial_{-} X^{-}\right)\right] \\
& \left.+w_{i}\left[P_{i}+\left(\frac{\lambda T}{2}\right)\left(\partial_{-} X^{i}\right)\right]\right]
\end{aligned}
$$

Hamiltons equations obtained from the total Hamiltonian $H_{4}^{T}=\int \mathcal{H}_{4}^{T} \mathrm{~d} \sigma^{-}$are the equations of motion that preserve the constraints of the theory in the course of time. The matrix of the Poisson brackets of these above constraints is seen to be singular implying that the corresponding set of constraints is first-class and that the corresponding theory is GI [13].

The above theory is indeed seen to possess three local gauge symmetries given by the 2D WSRI and the WI [13] and the theory could be quantized under appropriate guage-fixing. To study the Hamiltonian and path integral formulations of the theory under gauge-fixing, we could e.g., choose the gauge: $\eta=B \approx 0$. corresponding to this gauge choice the total set of 28 constraints of the theory becomes [13]:

$$
\begin{aligned}
& \phi_{1}=\eta=B \approx 0 \\
& \phi_{2}=\psi_{1}=\Pi_{B} \approx 0 \\
& \phi_{3}=\psi_{2}=\left[P^{+}+\frac{\lambda T}{2}\left(\partial_{-} X^{+}\right)\right] \approx 0 \\
& \phi_{4}=\psi_{3}=\left[P^{-}+\frac{\lambda T}{2}\left(\partial_{-} X^{-}\right)\right] \approx 0 \\
& \phi_{i}=\psi_{i}=\left[P_{i}+\frac{\lambda T}{2}\left(\partial_{-} X^{i}\right)\right] \approx 0, i=2,3, \cdots, 25
\end{aligned}
$$

The matrix of the Poisson brackets of the above 28 constraints is seen to be nonsingular implying that the corresponding set of constraints is second-class. Now following the Dirac quantization procedure in the Hamiltonian formulation, the nonvanishing ELCWST CR's of the theory under the gauge-fixing $\eta=B \approx 0$ are obtained as [13]:

$$
\left[X^{+}, P^{-}\right]=\left[X^{-}, P^{+}\right]=\left[X^{i}, P_{i}\right]=\frac{i}{2} \delta\left(\sigma-\sigma^{\prime}\right)
$$

$$
\begin{aligned}
& {\left[X^{-}, X^{+}\right]=\left[X^{i}, X^{i}\right]=\frac{i}{2 \lambda T} \epsilon\left(\sigma-\sigma^{\prime}\right)} \\
& {\left[P^{+}, P^{-}\right]=\left[P_{i}, P_{i}\right]=\frac{-i T \lambda}{4} \partial_{-} \delta\left(\sigma-\sigma^{\prime}\right)}
\end{aligned}
$$

For further details of the Hamiltonian and path integral formulations of the above theory, we refer to our earlier work [13].

\section{CGFPD1BA in the Presence of a Scalar Axion Field $C$ and an $U(1)$ Gauge Field $A^{\mu}$}

In this section, we study the IFQ and LFQ of the CGFPD1BA in the presence of a $U(1)$ gauge field $A_{\alpha}\left(\equiv A_{\alpha}(\tau, \sigma)\right)$ and a constant scalar axion field $C(\equiv C(\tau, \sigma)) \quad[19,20]$. We find that the CGFPD1BA describing a GNI theory (being a gauge-fixed theory) is seen to describe a GI theory when considered in the presence of above background fields.

We also find that the $U(1)$ gauge field $A_{\alpha}(\tau, \sigma)$ and the constant scalar axion field $C(\tau, \sigma)$ are both seen to behave like the Wess-Zumino (WZ) fields $[19,20]$ and the term involving these fields in the action is seen to behave like a WZ term for the CGFPD1BA $[13,19,20]$. Here the field $A_{\alpha}$ is a scalar field in the target space and a vector field in the WS space and the axion field $C$ is a constant scalar field in both the target space as well as in the WS space $[19,20]$.

We find that the resulting theory obtained in the above manner describes a GI system respecting the usual string gauge symmetries defined by the 2D WSRI and the WI. It is seen that the axion field $C$ and the $U(1)$ gauge field $A_{\alpha}$, in the resulting theory behave like the WZ fields and the term involving these fields behaves like a WZ term for the CGFPD1BA [19].

The situation in the present case is seen to be exactly analogous to a theory where one considers the CGFPD$1 \mathrm{BA}$ in the presence of a 2-form gauge field $B_{\alpha \beta}$ as studied by us in our earlier work [13], where the field $B_{\alpha \beta}$ behaves like a WZ field and the term involving this field behaves like a WZ term for the CGFPD1BA [13].

The CGFPD1BA in the presence of a constant background scalar axion field $C$ and an $U(1)$ gauge field $A_{\alpha}$ is defined by $[1-8,19,20]$ :

$$
\begin{aligned}
& S_{A}^{I}=\int \mathcal{L}_{A}^{I} \mathrm{~d}^{2} \sigma \\
& \mathcal{L}_{A}^{I}=\left(\mathcal{L}^{C}+\mathcal{L}^{A}\right) \\
& \mathcal{L}^{C}=\left(\lambda \mathcal{L}^{N}\right)=\left(\frac{-T}{2}\right)\left(\lambda \partial^{\beta} X^{\mu} \partial_{\beta} X_{\mu}\right) \\
& \mathcal{L}^{A}=\left(\frac{-T}{2}\right)\left(-\Lambda C \varepsilon^{\alpha \beta} F_{\alpha \beta}\right) \\
& \lambda=\sqrt{\left(1+\Lambda^{2}\right)}, \Lambda=\text { constant }
\end{aligned}
$$




$$
\begin{aligned}
& \varepsilon^{\alpha \beta}=\left(\begin{array}{ll}
0 & 1 \\
-1 & 0
\end{array}\right), F_{\alpha \beta}=\left(\partial_{\alpha} A_{\beta}-\partial_{\beta} A_{\alpha}\right) \\
& f=F_{01}=-F_{01}(I F Q) \\
& f=F_{+-}=-F_{-+}(L F Q) \\
& \alpha, \beta=0,1, \mu=0,1, i, i=2,3, \cdots, 25(I F Q) \\
& \alpha, \beta=+,-, \mu=+,-, i, i=2,3, \cdots, 25(L F Q)
\end{aligned}
$$

\subsection{Instant-Form Quantization}

In this section, we study the IF Hamiltonian and path integral quantization of the above theory using the EWST framework, on the hyperplanes defined by the WS-time $\sigma^{0}=\tau=$ constant . The IF action reads:

$$
S_{5}=\int \mathcal{L}_{5} \mathrm{~d} \tau \mathrm{d} \sigma, \mathcal{L}_{5}=\left[-\frac{\lambda T}{2}\left(\left(X^{\prime}\right)^{2}-(\dot{X})^{2}\right)+\Lambda T C f\right]
$$

Overdots and primes denote derivatives with respect to $\tau$ and $\sigma$ respectively. The canonical momenta obtained are:

$$
\begin{aligned}
& P^{\mu}:=\frac{\partial \mathcal{L}_{5}}{\partial\left(\partial_{\tau} X_{\mu}\right)}=\left[\lambda T \dot{X}^{\mu}\right] \\
& \Pi_{c}:=\frac{\partial \mathcal{L}_{5}}{\partial\left(\partial_{\tau} C\right)}=0 \\
& \Pi^{0}:=\frac{\partial \mathcal{L}_{5}}{\partial\left(\partial_{\tau} A_{0}\right)}=0 \\
& E\left(\equiv \Pi^{1}\right):=\frac{\partial \mathcal{L}_{5}}{\partial\left(\partial_{\tau} A_{1}\right)}=\Lambda T C
\end{aligned}
$$

where $P^{\mu}, \Pi^{0}, E\left(\equiv \Pi^{1}\right)$ and $\Pi_{c}$ are the canonical momenta conjugate respectively to $X_{\mu}, A_{0}, A_{1}$ and $C$. The theory is thus seen to possess three primary constraints:

$$
\begin{aligned}
& \Psi_{1}=\Pi^{0} \approx 0 \\
& \Psi_{2}=(E-\Lambda T C) \approx 0 \\
& \Psi_{3}=\Pi_{c} \approx 0
\end{aligned}
$$

Canonical Hamiltonian density corresponding to above Lagrangian density is:

$$
\begin{aligned}
\mathcal{H}_{5}^{c}= & {\left[P^{\mu}\left(\partial_{\tau} X_{\mu}\right)+\Pi^{0}\left(\partial_{\tau} A_{0}\right)\right.} \\
& \left.+E\left(\partial_{\tau} A_{1}\right)+\Pi_{c}\left(\partial_{\tau} C\right)-\mathcal{L}_{5}\right] \\
\mathcal{H}_{5}^{c}= & {\left[\frac{1}{2 \lambda T} P^{\mu} P_{\mu}+\frac{\lambda T}{2}\left(X^{\prime}\right)^{2}+\Lambda T C A_{0}^{\prime}\right] }
\end{aligned}
$$

After incorporating the primary constraints of the theory in the canonical Hamiltonian density with the help of
Lagrange multiplier fields $u(\tau, \sigma), v((\tau, \sigma))$ and $w(\tau, \sigma)$ (treated as dynamical) the total Hamiltonian density of the theory becomes:

$$
\begin{aligned}
\mathcal{H}_{5}^{T} & =\left[\mathcal{H}_{5}^{c}+u \Psi_{1}+u \Psi_{2}+w \Psi_{3}\right] \\
\mathcal{H}_{5}^{T} & =\left[\frac{1}{2 \lambda T} P^{\mu} P_{\mu}+\frac{\lambda T}{2}\left(X^{\prime}\right)^{2}+\Lambda T C A_{0}^{\prime}\right. \\
& \left.+\Pi^{0} u+(E-\Lambda T C) v+\Pi_{c} w\right]
\end{aligned}
$$

Momenta canonically conjugate to $u$, $v$ and $w$ are denoted respectively by $p_{u}, p_{v}$ and $p_{w}$. Hamiltons equations obtained from the total Hamiltonian $H_{5}^{T}=\int \mathcal{H}_{5}^{T} \mathrm{~d} \sigma$, for the closed string with periodic boundary conditions (BC's) e.g., are:

$$
\begin{aligned}
& \partial_{\tau} X^{\mu}=\frac{\partial H_{5}^{T}}{\partial P_{\mu}}=\left[\frac{1}{\lambda T}\right] P^{\mu}, \\
& -\partial_{\tau} P^{\mu}=\frac{\partial H_{5}^{T}}{\partial X_{\mu}}=(-\lambda T) X^{\prime \mu} \\
& \partial_{\tau} C=\frac{\partial H_{5}^{T}}{\partial \Pi_{C}}=w,-\partial_{\tau} \Pi_{C}=\frac{\partial H_{5}^{T}}{\partial C}=\Lambda T\left(A_{0}^{\prime}-v\right) \\
& \partial_{\tau} A_{0}=\frac{\partial H_{5}^{T}}{\partial \Pi^{0}}=u,-\partial_{\tau} \Pi^{0}=\frac{\partial H_{5}^{T}}{\partial A_{0}}=0 \\
& \partial_{\tau} A_{1}=\frac{\partial H_{5}^{T}}{\partial E}=v,-\partial_{\tau} E=\frac{\partial H_{5}^{T}}{\partial A_{1}}=0 \\
& \partial_{\tau} u=\frac{\partial H_{5}^{T}}{\partial p_{u}}=0,-\partial_{\tau} p_{u}=\frac{\partial H_{5}^{T}}{\partial u}=\Pi^{0} \\
& \partial_{\tau} v=\frac{\partial H_{5}^{T}}{\partial p_{v}}=0,-\partial_{\tau} p_{v}=\frac{\partial H_{5}^{T}}{\partial v}=(E-\Lambda T C) \\
& \partial_{\tau} w=\frac{\partial H_{5}^{T}}{\partial p_{w}}=0,-\partial_{\tau} p_{w}=\frac{\partial H_{5}^{T}}{\partial w}=\Pi_{C}
\end{aligned}
$$

These are the equations of motion of the theory that preserve the constraints of the theory in the course of time. Demanding that the primary constraints of the theory be preserved in the course of time one does not get any further constraints. The theory is thus seen to posses only three constraint $\Psi_{1}, \Psi_{2}$ and $\Psi_{3}$.

Matrix of the Poission brackets of these constraints is seen to be singular implying that the constraints form a set of first-class constraints and that the theory is GI (and consequently gauge-nonanomalous). It is indeed seen to posses three local gauge symmetries given by the $2 \mathrm{D}$ WSRI and the WI defined by:

$$
X^{\mu} \rightarrow \tilde{X}^{\mu}=\left[X^{\mu}+\delta X^{\mu}\right]
$$




$$
\begin{aligned}
& \delta X^{\mu}=\left[\zeta^{\alpha}\left(\partial_{\alpha} X^{\mu}\right)\right] \\
& h^{\alpha \beta} \rightarrow \tilde{h}^{\alpha \beta}=\left[h^{\alpha \beta}+\delta h^{\alpha \beta}\right] \\
& \delta h^{\alpha \beta}=\left[\zeta^{\gamma} \partial_{\gamma} h^{\alpha \beta}-\partial_{\gamma} \zeta^{\alpha} h^{\gamma \beta}-\partial_{\gamma} \zeta^{\beta} h^{\alpha \gamma}\right] \\
& A_{\beta} \rightarrow \tilde{A}_{\beta}=\left[A_{\beta}+\delta A_{\beta}\right] \\
& \delta A_{\beta}=\left[\zeta^{\alpha} \partial_{\alpha} A_{\beta}\right] \\
& C \rightarrow \tilde{C}_{\alpha}=[C+\delta C] \\
& \delta C=\left[\zeta^{\alpha} \partial_{\alpha} C\right] \\
& h_{\alpha \beta} \rightarrow\left[\Omega h_{\alpha \beta}\right], \Omega=\exp (2 \omega)
\end{aligned}
$$

The first order Lagrangian density of the theory is:

$$
\begin{aligned}
\mathcal{L}_{5}^{I O}= & {\left[P^{\mu}\left(\partial_{\tau} X_{\mu}\right)+\Pi^{0}\left(\partial_{\tau} A_{0}\right)+E\left(\partial_{\tau} A_{1}\right)+\Pi_{c}\left(\partial_{\tau} c\right)\right.} \\
& \left.+p_{u}\left(\partial_{\tau} u\right)+p_{v}\left(\partial_{\tau} v\right)+p_{w}\left(\partial_{\tau} w\right)-\mathcal{H}_{5}^{T}\right] \\
\mathcal{L}_{5}^{I O} & =\left[\frac{1}{2 \lambda T} P^{\mu} P_{\mu}+\frac{\lambda T}{2}\left(X^{\prime}\right)^{2}+\Lambda T C f\right]
\end{aligned}
$$

The theory could be quantized under appropriate gauge-fixing. To study the Hamiltonian and path integral formulations of this theory under gauge-fixing, we could choose e.g., the gauge:

$$
\zeta=A_{0} \approx 0
$$

Corresponding to this choice of gauge the total set of constraints of the theory under which the quantization of the theory could e.g., be studied becomes:

$$
\begin{aligned}
& \xi_{1}=\Psi_{1}=\Pi^{0} \approx 0 \\
& \xi_{2}=\Psi_{2}=(E-\Lambda T C) \approx 0 \\
& \xi_{3}=\Psi_{3}=\Pi_{c} \approx 0 \\
& \xi_{4}=\zeta=A_{0} \approx 0
\end{aligned}
$$

We now calculate the matrix $M_{\alpha \beta}\left(:=\left\{\Psi_{\alpha}, \Psi_{\beta}\right\}_{P B}\right)$ of the Poisson brackets of the constraints $\Psi_{i}$. The nonvanishing elements of the matrix $M_{\alpha \beta}$ are obtained as:

$$
\Lambda T M_{14}=-\Lambda T M_{41}=M_{23}=-M_{32}=[-\Lambda T] \delta\left(\sigma-\sigma^{\prime}\right)(44)
$$

The matrix $M_{\alpha \beta}$ is seen to be nonsingular implying that the corresponding set of constraints is a set of second-class constraints. The determinant of the matrix $M_{\alpha \beta}$ is given by:

$$
\left[\left\|\operatorname{det}\left(M_{\alpha \beta}\right)\right\|\right]^{1 / 2}=\Lambda T \delta^{2}\left(\sigma-\sigma^{\prime}\right)
$$

and the nonvanishing elements of the inverse of the matrix $M_{\alpha \beta}$ (i.e., the elements of the matrix $\left(M^{-1}\right)_{\alpha \beta}$ ) are obtained as:

$$
\begin{aligned}
\begin{aligned}
&\left(M^{-1}\right)_{14}=-\left(M^{-1}\right)_{41}=\Lambda T\left(M^{-1}\right)_{23} \\
&=-\Lambda T\left(M^{-1}\right)_{32}=\delta\left(\sigma-\sigma^{\prime}\right) \\
& \int M\left(\sigma, \sigma^{\prime \prime}\right) M^{-1}\left(\sigma^{\prime \prime}, \sigma^{\prime}\right) \mathrm{d} \sigma^{\prime \prime}=\mathbf{1}_{4 \times 4} \delta\left(\sigma-\sigma^{\prime}\right)
\end{aligned}
\end{aligned}
$$

Following the Dirac quantization procedure in the Hamiltonian formulation, the nonvanishing EWST CR's of the theory under the gauge $\zeta=A_{0} \approx 0$ (with the arguments being suppressed) are obtained as:

$$
\left[X^{\mu}(\sigma, \tau), P_{\mu}\left(\sigma^{\prime}, \tau\right)\right]=i \eta^{\mu v} \delta\left(\sigma-\sigma^{\prime}\right)
$$

In the path integral formulation, the transition to the quantum theory, is, however, made by writing the vacuum to vacuum transition amplitude called the generating functional $Z_{1}\left[J_{i}\right]$ of the theory under GFC $\zeta$ in the presence of external sources $J_{i}$ as follows:

$$
\begin{aligned}
Z_{1}\left[J_{i}\right] & :=\int[\mathrm{d} \mu] \exp \left[i \int \mathrm { d } ^ { 2 } \sigma \left[J_{i} \Phi^{i}\right.\right. \\
& \left.\left.+\frac{1}{2 \lambda T} P^{\mu} P_{\mu}+\frac{\lambda T}{2}\left(X^{\prime}\right)^{2}+\Lambda T C f\right]\right]
\end{aligned}
$$

where the phase space variables of the theory are $\Phi^{i} \equiv\left(X^{\mu}, A_{0}, A_{1}, C, u, v, w\right)$ with the corresponding respective canonical conjugate momenta:

$$
\Pi_{i} \equiv\left(P_{\mu}, \Pi^{0}, E, \Pi_{c}, p_{u}, p_{v}, p_{w}\right) .
$$

The functional measure $[\mathrm{d} \mu]$ of the generating functional $Z_{1}\left[J_{i}\right]$ under the GFC $\zeta$ is obtained as:

$$
\begin{aligned}
{[\mathrm{d} \mu]=} & {\left[\Lambda T \delta^{2}\left(\sigma-\sigma^{\prime}\right)\right]\left[\mathrm{d} X^{\mu}\right]\left[\mathrm{d} A_{0}\right]\left[\mathrm{d} A_{1}\right] } \\
& \cdot[\mathrm{d} C][\mathrm{d} u][\mathrm{d} v][\mathrm{d} w]\left[\mathrm{d} P_{\mu}\right]\left[\mathrm{d} \Pi^{0}\right][\mathrm{d} E] \\
& \cdot\left[\mathrm{d} \Pi_{c}\right]\left[\mathrm{d} p_{u}\right]\left[\mathrm{d} p_{v}\right]\left[\mathrm{d} p_{w}\right] \\
& \times \delta\left[\left(\Pi^{0}\right) \approx 0\right] \delta\left[\left(\Pi_{c}\right) \approx 0\right] \delta\left[\left(A_{0}\right) \approx 0\right] \\
& \cdot \delta[(E-\Lambda T C) \approx 0]
\end{aligned}
$$

The Hamiltonian and path integral quantization of the above theory under the GFC $\zeta=A_{0} \approx 0$ is now complete.

\subsection{Light-Front Quantization}

In LFQ, the action of the theory reads:

$$
\begin{aligned}
S_{6} & =\int \mathcal{L}_{6} \mathrm{~d} \sigma^{+} \mathrm{d} \sigma^{-} \\
\mathcal{L}_{6} & =\left[\frac{-\lambda T}{2}\right]\left[\left(\partial_{+} X^{+}\right)\left(\partial_{-} X^{-}\right)\right. \\
& \left.+\left(\partial_{+} X^{-}\right)\left(\partial_{-} X^{+}\right)+\left(\partial_{+} X^{i}\right)\left(\partial_{-} X^{i}\right)+\Lambda T C f\right]
\end{aligned}
$$

In the following we study the LF Hamiltonian and path integral formulations of the above action. The canonical 
momenta $P^{+}, P^{-}, P_{i}, \Pi_{c}, \Pi^{+}$and $\Pi^{-}$conjugate respectively to $X^{-}, X^{+}, X_{i}, C, A^{-}$and $A^{+}$are obtained as:

$$
\begin{gathered}
\Pi^{+}=\frac{\partial \mathcal{L}_{6}}{\partial\left(\partial_{+} A^{-}\right)}=0 \\
\Pi^{-}=\frac{\partial \mathcal{L}_{6}}{\partial\left(\partial_{+} A^{+}\right)}=\Lambda T C \\
\Pi_{c}:=\frac{\partial \mathcal{L}_{6}}{\partial\left(\partial_{+} C\right)}=0 \\
P^{+}:=\frac{\partial \mathcal{L}_{6}}{\partial\left(\partial_{+} X^{-}\right)}=-\frac{\lambda T}{2}\left(\partial_{-} X^{+}\right) \\
P^{-}:=\frac{\partial \mathcal{L}_{6}}{\partial\left(\partial_{+} X^{+}\right)}=-\frac{\lambda T}{2}\left(\partial_{-} X^{-}\right) \\
P_{i}:=\frac{\partial \mathcal{L}_{6}}{\partial\left(\partial_{+} X^{i}\right)}=-\frac{\lambda T}{2}\left(\partial_{-} X^{i}\right), \\
i=2,3, \cdots, 25
\end{gathered}
$$

Above equations however, imply that the theory possesses 29 primary constraints:

$$
\begin{aligned}
& \chi_{1}=\Pi^{+} \approx 0 \\
& \chi_{2}=\left(\Pi^{-}-\Lambda T C\right) \approx 0 \\
& \chi_{3}=\Pi_{c} \approx 0 \\
& \chi_{4}=\left[P^{+}+\frac{\lambda T}{2}\left(\partial_{-} X^{+}\right)\right] \approx 0 \\
& \chi_{5}=\left[P^{-}+\frac{\lambda T}{2}\left(\partial_{-} X^{-}\right)\right] \approx 0 \\
& \chi_{i}=\left[P_{i}+\frac{\lambda T}{2}\left(\partial_{-} X^{i}\right)\right] \approx 0, \\
& i=2,3, \cdots, 25
\end{aligned}
$$

Canonical Hamiltonian density of this theory is:

$$
\begin{aligned}
\mathcal{H}_{6}^{c}= & {\left[P^{+}\left(\partial_{+} X^{-}\right)+P^{-}\left(\partial_{+} X^{+}\right)+P_{i}\left(\partial_{+} X^{i}\right)\right.} \\
& \left.+\Pi^{+}\left(\partial_{+} A^{-}\right)+\Pi^{-}\left(\partial_{+} A^{+}\right)+\Pi_{c}\left(\partial_{+} C\right)-\mathcal{L}_{6}\right] \\
\mathcal{H}_{6}^{c}= & {\left[\Lambda T C\left(\partial_{-} A^{-}\right)\right] }
\end{aligned}
$$

After including the above 29 primary constraints in the canonical Hamiltonian density $\mathcal{H}_{6}^{c}$ with the help of Lagrange multiplier fields $v_{1}\left(\sigma^{+}, \sigma^{-}\right), v_{2}\left(\sigma^{+}, \sigma^{-}\right)$, $v_{3}\left(\sigma^{+}, \sigma^{-}\right), \quad v_{4}\left(\sigma^{+}, \sigma^{-}\right), v_{5}\left(\sigma^{+}, \sigma^{-}\right)$and $v_{i}\left(\sigma^{+}, \sigma^{-}\right)$ (which we treat as dynamical), the total Hamiltonian density of the theory could be written as:

$$
\mathcal{H}_{6}^{T}=\left[\mathcal{H}_{6}^{c}+s_{1} \chi_{1}+s_{2} \chi_{2}+s_{3} \chi_{3}+u \chi_{4}+v \chi_{5}+w_{i} \chi_{i}\right](54 \mathrm{a})
$$

$$
\begin{aligned}
\mathcal{H}_{6}^{T} & =\left[\Lambda T C\left(\partial_{-} A^{-}\right)+u\left[P^{+}+\left(\frac{\lambda T}{2}\right)\left(\partial_{-} X^{+}\right)\right]\right. \\
& +v\left[P^{-}+\left(\frac{\lambda T}{2}\right)\left(\partial_{-} X^{-}\right)\right]+w_{i}\left[P_{i}+\left(\frac{\lambda T}{2}\right)\left(\partial_{-} X^{i}\right)\right] \\
& \left.+s_{1} \Pi^{+}+s_{2}\left(\Pi^{-}-\Lambda T C\right)+s_{3} \Pi_{c}\right]
\end{aligned}
$$

The Hamiltons equations of motion of the theory that preserve the constraints of the theory in the course of time obtained from the total Hamiltonian:

$$
H_{6}^{T}=\int \mathcal{H}_{6}^{T} \mathrm{~d} \sigma^{-}
$$

e.g., for the closed strings with periodic BC's are obtained as:

$$
\begin{aligned}
& \partial_{+} X^{-}=\frac{\partial H_{6}^{T}}{\partial P^{+}}=u,-\partial_{+} P^{+}=\frac{\partial H_{6}^{T}}{\partial X^{-}}=\left[-\frac{\lambda T}{2}\right]\left(\partial_{-} v\right) \\
& \partial_{+} X^{+}=\frac{\partial H_{6}^{T}}{\partial P^{-}}=v,-\partial_{+} P^{-}=\frac{\partial H_{6}^{T}}{\partial X^{+}}=\left[-\frac{\lambda T}{2}\right]\left(\partial_{-} u\right) \\
& \partial_{+} X^{i}=\frac{\partial H_{6}^{T}}{\partial P_{i}}=w_{i},-\partial_{+} P_{i}=\frac{\partial H_{6}^{T}}{\partial X^{i}}=\left[-\frac{\lambda T}{2}\right]\left(\partial_{-} w_{i}\right) \\
& \partial_{+} u=\frac{\partial H_{6}^{T}}{\partial p_{u}}=0,-\partial_{+} p_{u}=\frac{\partial H_{6}^{T}}{\partial u}=\left(P^{+}+\frac{\lambda T}{2} \partial_{-} X^{+}\right) \\
& \partial_{+} v=\frac{\partial H_{6}^{T}}{\partial p_{v}}=0,-\partial_{+} p_{v}=\frac{\partial H_{6}^{T}}{\partial v}=\left(P^{-}+\frac{\lambda T}{2} \partial_{-} X^{-}\right) \\
& \partial_{+} w_{i}=\frac{\partial H_{6}^{T}}{\partial p_{w_{i}}}=0,-\partial_{+} p_{w_{i}}=\frac{\partial H_{6}^{T}}{\partial w_{i}}=\left(P_{i}+\frac{\lambda T}{2} \partial_{-} X^{i}\right) \\
& \partial_{+} C=\frac{\partial H_{6}^{T}}{\partial \Pi_{C}}=s_{3},-\partial_{+} \Pi_{C}=\frac{\partial H_{6}^{T}}{\partial C}=\Lambda T\left(\partial_{-} A^{-}-s_{2}\right) \\
& \partial_{+} A^{+}=\frac{\partial H_{6}^{T}}{\partial \Pi^{-}}=s_{2},-\partial_{+} \Pi^{-}=\frac{\partial H_{6}^{T}}{\partial A^{+}}=0 \\
& \partial_{+} A^{-}=\frac{\partial H_{6}^{T}}{\partial \Pi^{+}}=s_{1},-\partial_{+} \Pi^{+}=\frac{\partial H_{6}^{T}}{\partial A^{-}}=0 \\
& \partial_{+} s_{1}=\frac{\partial H_{6}^{T}}{\partial p_{s_{1}}}=0,-\partial_{+} p_{s_{1}}=\frac{\partial H_{6}^{T}}{\partial s_{1}}=\Pi^{+} \\
& \partial_{+} s_{2}=\frac{\partial H_{6}^{T}}{\partial p_{s_{2}}}=0,-\partial_{+} p_{s_{2}}=\frac{\partial H_{6}^{T}}{\partial s_{2}}=\left(\Pi^{-}-\Lambda T C\right) \\
& \partial_{+} s_{3}=\frac{\partial H_{6}^{T}}{\partial p_{s_{3}}}=0,-\partial_{+} p_{s_{3}}=\frac{\partial H_{6}^{T}}{\partial s_{3}}=\Pi_{C}
\end{aligned}
$$

Demanding that the primary constraints of the theory be preserved in the course of time one does not get any secondary constraints. The theory is thus seen to possess 
only 29 constraints: $\chi_{1}, \chi_{2}, \chi_{3}, \chi_{4}, \chi_{5}$ and $\chi_{i}$. Further the matrix of the Poisson brackets of these 29 constraints among themselves is easily seen to be singular, implying that the set of these 29 constarints is first-class. This in turn implies that the theory is GI (and consequently gauge anomalous). The theory is indeed seen to possess three local gauge symmetries given by the 2D WSRI and the WI defined by Equation (40). The theory could now be quantized under appropriate guage-fixing.

The first-order Lagrangian density of the theory is:

$$
\begin{aligned}
\mathcal{L}_{6}^{I O}= & {\left[\Pi_{c}\left(\partial_{+} C\right)+P^{+}\left(\partial_{+} X^{-}\right)+P^{-}\left(\partial_{+} X^{+}\right)\right.} \\
& +P_{i}\left(\partial_{+} X^{i}\right)+\Pi^{+}\left(\partial_{+} A^{-}\right)+\Pi^{-}\left(\partial_{+} A^{+}\right) \\
& +p_{s_{1}}\left(\partial_{+} s_{1}\right)+p_{s_{2}}\left(\partial_{+} s_{2}\right)+p_{s_{3}}\left(\partial_{+} s_{3}\right) \\
& \left.+p_{u}\left(\partial_{+} u\right)+p_{v}\left(\partial_{+} v\right)+p_{w_{i}}\left(\partial_{+} w_{i}\right)-\mathcal{H}_{6}^{T}\right] \\
\mathcal{L}_{6}^{I O}= & {\left[\Lambda T C\left(s_{2}-\partial_{-} A^{-}\right)\right.} \\
& \left.+\frac{\lambda T}{2}\left[u\left(\partial_{-} X^{+}\right)+v\left(\partial_{-} X^{-}\right)+w_{i}\left(\partial_{-} X^{i}\right)\right]\right]
\end{aligned}
$$

To study the Hamiltonian and path integral formulations of the theory under gauge-fixing, we could e.g., choose the gauge:

$$
\theta=A^{-} \approx 0
$$

corresponding to this gauge choice, the total set of constraints of the theory under which the quantization of the theory could be studied becomes:

$$
\begin{aligned}
& \eta_{1}=\chi_{1}=\Pi^{+} \approx 0 \\
& \eta_{2}=\chi_{2}=\left(\Pi^{-}-\Lambda T C\right) \approx 0 \\
& \eta_{3}=\chi_{3}=\Pi_{c} \approx 0 \\
& \eta_{4}=\theta=A^{-} \approx 0 \\
& \eta_{5}=\chi_{4}=\left[P^{+}+\frac{\lambda T}{2}\left(\partial_{-} X^{+}\right)\right] \approx 0 \\
& \eta_{6}=\chi_{5}=\left[P^{-}+\frac{\lambda T}{2}\left(\partial_{-} X^{-}\right)\right] \approx 0 \\
& \eta_{i}=\chi_{i}=\left[P_{i}+\frac{\lambda T}{2}\left(\partial_{-} X^{i}\right)\right] \approx 0, i=2,3, \cdots, 25
\end{aligned}
$$

We now calculate the matrix

$$
R_{\alpha \beta}\left(\sigma, \sigma^{\prime}\right)\left(:=\left\{\eta_{\alpha}(\sigma), \eta_{\beta}\left(\sigma^{\prime}\right)\right\}_{\mathrm{PB}}\right)
$$

of the Poisson brackets of these above 30 constraints. The nonvanishing elements of the matrix $R_{\alpha \beta}$ are obtained as:

$$
R_{56}=+R_{65}=R_{i i}=(\lambda T) \partial_{-} \delta\left(\sigma-\sigma^{\prime}\right), i=2,3, \cdots, 25(60 \mathrm{a})
$$

$$
\Lambda T R_{14}=-\Lambda T R_{41}=R_{23}=-R_{32}=(-\Lambda T) \delta\left(\sigma-\sigma^{\prime}\right)
$$

The matrix $R_{\alpha \beta}$ is seen to be nonsingular implying that the corresponding set of these 30 constraints is second-class. The determinant of the matrix $R_{\alpha \beta}$ is given by

$$
\begin{aligned}
& {\left[\left\|\operatorname{det}\left(R_{\alpha \beta}\right)\right\|\right]^{1 / 2}} \\
& =\left[\left(\Lambda T \delta^{2}\left(\sigma-\sigma^{\prime}\right)\right)\left(\lambda T \partial_{-} \delta\left(\sigma-\sigma^{\prime}\right)\right)^{13}\right]
\end{aligned}
$$

Nonvanishing elements of the inverse of this matrix $R_{\alpha \beta}$ (i.e. the elements of the matrix $\left.\left(R^{-1}\right)_{\alpha \beta}\right)$ are:

$$
\begin{aligned}
& \left(R^{-1}\right)_{56}=\left(R^{-1}\right)_{65}=\left(R^{-1}\right)_{i i}=\frac{1}{2 \lambda T} \epsilon\left(\sigma-\sigma^{\prime}\right), \\
& \begin{aligned}
& i=2,3, \cdots, 25 \\
&\left(R^{-1}\right)_{14}=-\left(R^{-1}\right)_{41}=\Lambda T\left(R^{-1}\right)_{23} \\
&=-\Lambda T\left(R^{-1}\right)_{32}=\delta\left(\sigma-\sigma^{\prime}\right)
\end{aligned}
\end{aligned}
$$

with

$$
\int R\left(\sigma, \sigma^{\prime \prime}\right) R^{-1}\left(\sigma^{\prime \prime}, \sigma^{\prime}\right) \mathrm{d} \sigma^{\prime \prime}=\mathbf{1}_{30 \times 30} \delta\left(\sigma-\sigma^{\prime}\right)
$$

Finally, following the Dirac quantization procedure in the Hamiltonian formulation, the nonvanishing ELCWST commutation relations of this theory under the gauge $\theta=A^{-} \approx 0$ (with the arguments being supproted again) are obtained as:

$$
\begin{aligned}
& {\left[X^{+}, P^{-}\right]=\left[X^{-}, P^{+}\right]=\left[X^{i}, P_{i}\right]=[i / 2] \delta\left(\sigma-\sigma^{\prime}\right)} \\
& {\left[X^{-}, X^{+}\right]=\left[X^{i}, X^{i}\right]=[i /(2 \lambda T)] \epsilon\left(\sigma-\sigma^{\prime}\right)} \\
& {\left[P^{+}, P^{-}\right]=\left[P_{i}, P_{i}\right]=[-i T \lambda / 4] \partial_{-} \delta\left(\sigma-\sigma^{\prime}\right)} \\
& {\left[A^{+}, \Pi^{-}\right]=\Lambda T\left[A^{+}, C\right]=(i) \delta\left(\sigma-\sigma^{\prime}\right)}
\end{aligned}
$$

In the path integral formulation, the transition to the quantum theory, is, however, made by writing the vacuum to vacuum transition amplitude called the generating functional $Z_{2}\left[J_{i}\right]$ of the theory in the presence of external sources $J_{i}$ as follows:

$$
\begin{aligned}
& Z_{2}\left[J_{i}\right] \\
& :=\int[\mathrm{d} \mu] \exp \left[i \int \mathrm { d } \sigma ^ { + } \mathrm { d } \sigma ^ { - } \left[J_{i} \Phi^{i}+\Lambda T C\left(s_{2}-\partial_{-} A^{-}\right)\right.\right. \\
& \left.\left.+\left(\frac{\lambda T}{2}\right)\left[u\left(\partial_{-} X^{+}\right)+v\left(\partial_{-} X^{-}\right)+w_{i}\left(\partial_{-} X^{i}\right)\right]\right]\right]
\end{aligned}
$$

where the phase space variables of the theory are $\Phi^{i} \equiv\left(C, X^{+}, X^{-}, X^{i}, A^{+}, A^{-}, v_{1}, v_{2}, v_{3}, v_{4}, v_{5}, v_{6}\right)$ with the corresponding respective canonical conjugate momenta: $\Pi_{i} \equiv\left(\Pi_{C}, P^{-}, P^{+}, P_{i}, p_{v_{1}}, p_{v_{2}}, p_{v_{3}}, p_{v_{4}}, p_{v_{5}}, p_{v_{6}}\right)$. The functional measure $[\mathrm{d} \mu]$ of the generating functional 
$Z_{2}\left[J_{i}\right]$ is obtained as:

$$
\begin{aligned}
{[\mathrm{d} \mu] } & =\left(\Lambda T \delta^{2}\left(\sigma-\sigma^{\prime}\right)\right)\left(\lambda T \partial_{-} \delta\left(\sigma-\sigma^{\prime}\right)\right)^{13} \\
& \cdot[\mathrm{d} C]\left[\mathrm{d} X^{+}\right]\left[\mathrm{d} X^{-}\right]\left[\mathrm{d} X^{i}\right]\left[\mathrm{d} A^{+}\right]\left[\mathrm{d} A^{-}\right] \\
& \times\left[\mathrm{d} v_{1}\right]\left[\mathrm{d} v_{2}\right]\left[\mathrm{d} v_{3}\right]\left[\mathrm{d} v_{4}\right]\left[\mathrm{d} v_{5}\right]\left[\mathrm{d} v_{6}\right]\left[\mathrm{d} \Pi_{c}\right] \\
& \cdot\left[\mathrm{d} P^{-}\right]\left[\mathrm{d} P^{+}\right]\left[\mathrm{d} P_{i}\right]\left[\mathrm{d} \Pi^{+}\right]\left[\mathrm{d} \Pi^{-}\right] \\
& \times\left[\mathrm{d} p_{v_{1}}\right]\left[\mathrm{d} p_{v_{2}}\right]\left[\mathrm{d} p_{v_{3}}\right]\left[\mathrm{d} p_{v_{4}}\right]\left[\mathrm{d} p_{v_{5}}\right]\left[\mathrm{d} p_{v_{6}}\right] \\
& \cdot \delta\left[\left(P^{+}+\frac{\lambda T}{2} \partial_{-} X^{+}\right) \approx 0\right] \\
& \times \delta\left[\left(P^{-}+\frac{\lambda T}{2} \partial_{-} X^{-}\right) \approx 0\right] \\
& \cdot \Pi_{i}\left(\delta\left[\left(P_{i}+\frac{\lambda T}{2} \partial_{-} X^{i}\right) \approx 0\right]\right) \\
& \times \delta\left[\left(\Pi^{+}\right) \approx 0\right] \delta\left[\left(\Pi^{-}-\Lambda T C\right) \approx 0\right] \\
& \cdot \delta\left[\left(\Pi_{c}\right) \approx 0\right] \delta\left[\left(A^{-}\right) \approx 0\right]
\end{aligned}
$$

where $\Pi_{i}$ denotes the product of similar expressions for all $i=2,3, \cdots, 25$. The LF Hamiltonian and path integral quantization of the above theory is now complete.

\section{Summary and Discussion}

The Polyakov D1 brane action in a d-dimensional courved background $h_{\alpha \beta}$ defined by (Equation (1)) is GI and it possesses the well-known three local gauge symmetries given by the 2D WSRI and the WI defined by Equation (2).

However, when we study this action under the conformal gauge-fixing defined by Equation (3) to obtain the CGFPD1BA defined by the action $S^{N}$, we find that the CGFPD1BA given by $S^{N}$ is no longer GI and it describes a gauge anomalous (and GNI) theory and it also does not possess the usual local gauge symmetries defined by Equation (2) being a gauge-fixed theory. Hovever, this GNI theory when considered in the presence of a contant antisymmetric 2-form gauge field $B_{\alpha \beta}$ is seen to become a GI theory possessing the three local gauge symmetries defined by the 2D WSRI and the WI defined by Equation (24).

The 2-form gauge field $B_{\alpha \beta}$ in this case is seen to behave like a WZ field and the term involving this field is seen to behave like a WZ term for the CGFPD1BA which, in the absence of this term, is seen to possess a set of second-class constraints and consequently describe a GNI theory which does not respect the local gauge symmetries defined by the WSRI and WI given by Equation (2).

In our earlier work (cf. Section 3) [13], we have studied the IF and LF Hamiltonian and path integral formula- tions of this GI theory describing the CGFPD1BA in the presence of the constant antisymmetric 2-form gauge field have been studied under appropriate gauge choices in the abesence of BC's. The BC's could however be taken into account either by imposing them directly in the usual way for the open and closed strings separately in an appropriate manner or by considering them as the Dirac primary constraints [13-20,34] and study them accordingly.

In the present work [20], we have studied the IF and LF Hamiltonian and path integral formulations of the CGFPD1BA in the presence of a constant scalar axion field $C$ and an $U(1)$ gauge field $A_{\alpha}$ (cf. Section 4). We find that the scalar axion field $C$ and the $U(1)$ gauge field $A_{\alpha}$ are seen to behave like the WZ field and the term involving these fields is seen to behave like a WZ term for the CGFPD1BA, which in the absence of this term is seen to posess a set of second-class constraints and consequently describe a GNI theory which does not respect the local gauge symmetries defined by the WSRI and WI.

The situation in the present case, as pointed out in the foregoing, is analogous to the theory where one considerers the CGFPD1BA in the presence of the constant 2-form gauge field $B_{\alpha \beta}$, where $B_{\alpha \beta}$ which is a scalar in the target space and an antisymmetric tensor in the WS space, behaves like a WZ field and the term involving this field behaves like a WZ term for the CGFPD1BA [13]. The later theory has been studied by the present authors in Ref. [13] to which we refer the reader for further details [3].

The IF and FF Hamiltonian and path integral formulations of the GI theory describing the CGFPD1BA in the presence of the constant scalar axion field $C$ and the $U(1)$ gauge field $A_{\alpha}$ have been studied in this work under appropriate gauge choices in the abesence of BC's [34]. The BC's, however, could be taken into account in the usual manner either by imposing them directly in the usual way for the open and closed strings separately [1-8] or by considering them as the Dirac primary constraints, and study them accordingly [13-20,34].

In conclusion, the Polyakov D1 brane action in a d-dimensional courved background $h_{\alpha \beta}$ defined by $\tilde{S}$ is GI and it possesses the well-known three local string gauge symmetries. However, under conformal gaugefixing, the CGFPD1BA is no longer GI as expected and it also does not possess the local string gauge symmetries being a gauge-fixed theory. Hovever, this GNI theory when considered in the presence of a contant background scalar axiom field $C$ and an $U(1)$ gauge field $A_{\alpha}$ it is seen to become a GI theory possessing the three local string gauge symmetries.

The scalar axion field $C$ and the $U(1)$ gauge field $A_{\alpha}$ are seen to behave like the WZ fields and the term 
$\left(\mathcal{L}^{A}\right)$ involving these fields is seen to behave like a WZ term for the CGFPD1BA, which in the absence of this term is seen to posess a set of second-class constraints and consequently describes a GNI theory which does not respect the local string gauge symmetries. The situation in the present case is analogous to a theory where one considerers the CGFPD1BA in the presence of a constant 2-form gauge field $B_{\alpha \beta}$ which behaves like a WZ field and the term involving this field behaves like a WZ term for the CGFPD1BA.

\section{Acknowledgements}

Authors thank Prof. James Vary for several useful discussions and for his warm hospitality at the Iowa State University, Ames, Iowa, USA, where a part of this work was done.

\section{REFERENCES}

[1] D. Luest and S. Theisen, "Lectures in String Theory," Springer Verlag, Berlin, 1989.

[2] L. Brink and M. Henneaux, "Principles of String Theory,” Plenum Press, New York, 1988. doi:10.1007/978-1-4613-0909-3

[3] C. V. Johnson, "D-Brane Primer," Prepared for Theoretical Advanced Study Institute in Elementary Particle Physics (TASI 99): Strings, Branes, and Gravity, Boulder, Colorado, 31 May-25 June 1999, pp. 129-350. arXiv: hep-th/0007170

[4] M. Aganagic, J. Park, C. Popescu and J. Schwarz, "Dual D-Brane Actions,” Nuclear Physics B, Vol. 496, No. 1-2, 1997, pp. 215-230. doi:10.1016/S0550-3213(97)00257-5

[5] M. Abou Zeid and C. M. Hull, "Intrinsic Geometry of D-Branes,” Physics Letters B, Vol. 404, No. 3-4, 1997, pp. 264-270. doi:10.1016/S0370-2693(97)00570-4

[6] C. Schmidhuber, "D-Brane Actions," Nuclear Physics B, Vol. 467, No. 1-2, 1996, pp. 146-158. doi:10.1016/0550-3213(96)00092-2

[7] S. P. de Alwis and K. Sato, "D-Strings and F-Strings from String Loops,” Physical Review D, Vol. 53, No. 12, 1996, pp. 7187-7196. doi:10.1103/PhysRevD.53.7187

[8] A. A. Tseytlin, "Self Duality of Born-Infeld Action and Dirichlet Three-Brane of Type IIB Super String Theory," Nuclear Physics B, Vol. 469, No. 1-2, 1996, pp. 51-67. doi:10.1016/0550-3213(96)00173-3

[9] U. Kulshreshtha and D. S. Kulshreshtha, "Hamiltonian and Path Integral Formulations of the Dirac-Born-Infeld-Nambu-Goto D1 Brane Action with and without a Dilaton Field under Gauge-Fixing," European Physical Journal C, Vol. 29, No. 3, 2003, pp. 453-461. doi:10.1140/epjc/s2003-01239-8

[10] U. Kulshreshtha and D. S. Kulshreshtha, "Hamiltonian and Path Integral Formulations of the Nambu-Goto D1 Brane Action with and Without a Dilaton Field under Gauge-Fixing," International Journal of Theoretical Physics, Vol. 43, No. 12, 2004, pp. 2355-2369.

\section{doi:10.1007/s10773-004-7704-5}

[11] U. Kulshreshtha and D. S. Kulshreshtha, "Hamiltonian and Path Integral Formulations of the Born-InfeldNambu-Goto D1 Brane Action with and without a Dilaton Field under Gauge-Fixing," International Journal of Theoretical Physics, Vol. 44, No. 5, 2005, pp. 587-603. doi:10.1007/s10773-005-3985-6

[12] U. Kulshreshtha and D. S. Kulshreshtha, "Hamiltonian and Path Integral Quantization of the Conformally Gauge-Fixed Polyakov D1 Brane Action in the Presence of a Scalar Dilation Field," International Journal of Theoretical Physics, Vol. 48, No. 4, 2009, pp. 937-944. doi:10.1007/s10773-008-9866-Z

[13] U. Kulshreshtha and D. S. Kulshreshtha, "Conformally Gauge-Fixed Polyakov D1 Brane Action in the Presence of a 2-Form Gauge Field: The Instant-Form and FrontForm Hamiltonian and Path Integral Formulations," Physics Letters B, Vol. 555, No. 3-4, 2003, pp. 255-263. doi:10.1016/S0370-2693(03)00056-X

[14] D. S. Kulshreshtha, "Light-Front Quantization of the Polyakov D1 Brane Action with a Scalar Dilaton Field,” Invited Talk at the Light-Cone 2007: Relativistic Hadronic and Nuclear Physics (LC2007), Columbus, 14-18 May, 2007.

[15] D. S. Kulshreshtha, "Polyakov D1 Brane Action on the Light-Front," Invited Talk at the Light-Cone 2008: Relativistic Nuclear and Particle Physics (2008), Mulhouse, 7-11 July 2008.

[16] D. S. Kulshreshtha, "String Gauge Symmetries of the Light-Front Polyakov D1 Brane Action,” Invited Talk at the Light-Cone International Workshop on "Relativistic Hadronic and Particle Physics,” LC2010, Valencia, 14-18 June 2010.

[17] U. Kulshreshtha and D. S. Kulshreshtha, "Light-Front Hamiltonian and Path Integral Quantization of the Conformally Guage-Fixed Polyakov D1 Brane Action,” Journal of Modern Physics, Vol. 2, No. 5, 2011, pp. 335340. doi:10.4236/jmp.2011.25041

[18] U. Kulshreshtha and D. S. Kulshreshtha, "Light-Front Hamiltonian and Path Integral Formulations of the Conformally Gauge-Fixed Polyakov D1 Brane Action in the Presence of a Scalar Dilaton Field,” Journal of Modern Physics, Vol. 2, No.8, 2011, pp. 826-833. doi:10.4236/jmp.2011.28097

[19] U. Kulshreshtha and D. S. Kulshreshtha, "String Gauge Symmetries in the Conformally Gauge-Fixed Polyakov D1-Brane Action in the Presence of Background Gauge Fields," Journal of Modern Physics, Vol. 3, No. 1, 2012, pp. 110-115. doi:10.4236/jmp.2012.31015

[20] D. S. Kulshreshtha, "Light-Front Quantization of Conformally Gauge-Fixed Polyakov D1-Brane Action in the Presence of a Scalar Axion Field and an $U(1)$ Gauge Field,” Few Body Systems, Vol. 52, 2012, pp. 463-467. doi:10.1007/s00601-011-0279-9

[21] A. M. Dirac, “Generalized Hamiltonian Dynamics,” $\mathrm{Ca}$ nadian Journal of Mathematics, Vol. 2, 1950, pp. 129148. doi:10.4153/CJM-1950-012-1

[22] M. Henneaux and C. Teitleboim, "Quantization of Gauge 
Systems,” Princeton University Press, Princeton, 1992.

[23] P. Senjanovic, "Path Integral Quantization of Field Theories with Second-Class Constraints," Annals Physics, Vol. 100, No. 1-2, 1976, pp. 227-261.

[24] U. Kulshreshtha, "Hamiltonian, Path Integral and BRST Formulations of the Chern-Simons-Higgs Theory in the Broken Symmetry Phase,” Physica Scripta, Vol. 75, No. 6, 2007, pp. 795-802. doi:10.1088/0031-8949/75/6/009

[25] U. Kulshreshtha and D. S. Kulshreshtha, "Gauge-Invariant Reformulation of the Vector Schwinger Model with a Photon Mass Term and Its Hamiltonian, Path Integral and BRST Formulations," International Journal of Modern Physics A, Vol. 22, No. 32, 2007, pp. 6183-6201. doi:10.1142/S0217751X07038049

[26] P.A. M. Dirac, "Forms of Relativistic Dynamics," Reviews of Modern Physics, Vol. 21, No. 3, 1949, pp. 392399. doi:10.1103/RevModPhys.21.392

[27] S. J. Brodsky, H. C. Pauli and S. S. Pinsky, "Quantum Chromodynamics and Other Field Theories on the Light-Cone,” Physics Reports, Vol. 301, No. 4-6, 1998, pp. 299-486.

[28] U. Kulshreshtha, D. S. Kulshreshtha and J. P. Vary, "Light-Front Hamiltonian and Path Integral Formulations of the Large N scalar QCD $_{2}$," Physical Letters, Vol. 708, 2012, pp. 195-198.

[29] U. Kulshreshtha, "Light-Front Hamiltonian, Path Integral and BRST Formulations of the Nelsen-Olsen (Bogomol'nyi) Model in the Light-Cone Gauges," International
Journal of Theoretical Physics, Vol. 46, No. 10, 2007, pp. 2516-2530. doi:10.1007/s10773-007-9367-5

[30] U. Kulshreshtha, D. S. Kulshreshtha and J. P. Vary, "Light-Front Hamiltonian, Path Integral and BRST Formulations of the Chern-Simons-Higgs Theory under Appropriate Gauge-Fixing,” Physics Scripta, Vol. 82, No. 5, 2010, Article ID: 055101. doi:10.1088/0031-8949/82/05/055101

[31] U. Kulshreshtha, D. S. Kulshreshtha and J. P. Vary, "Light-Front Hamiltonian, Path Integral and BRST Formulations of the Chern-Simons Theory under Appropriate Gauge-Fixing," Journal of Modern Physics, Vol. 1, No. 6, 2010, pp. 385-392. doi:10.4236/jmp.2010.16055

[32] U. Kulshreshtha, D. S. Kulshreshtha and J. P. Vary, "Light-Front Hamiltonian, Path Integral and BRST Formulations of the Chern-Simons Theory under Appropriate Gauge-Fixing," Journal of Modern Physics, Vol. 1, No. 6. 2010, pp. 385-392.

[33] U. Kulshreshtha, D. S. Kulshreshtha and J. P. Vary, "Light-Front Hamiltonian, Path Integral and BRST Formulations of the Chern-Simons-Higgs Theory under Appropriate Gauge-Fixing,” Physics Scripta, Vol. 82, 2010, Article ID: 055101. doi:10.1088/0031-8949/82/05/055101

[34] M. M. Sheikh-Jabbari and A. Shirzad; "Boundary Conditions as Dirac Constraints,” The European Physical Journal C, Vol. 19, No. 2, 2001, pp. 383-390.

doi:10.1007/s100520100590 